Equipment was limited, and essentials such as scalpel blades and rubber gloves were often missing and had to be borrowed from the R.A.F. It is reliably reported that a certain surgeon on one occasion had to use scissors to make his skin incision for an abdominal operation. There was no anaesthetic machine. For head-and-neck surgery a Heath-Robinson arrangement of borrowed equipment was used. A catheter was attached to rubber tubing and this in turn to a funnel. On to a piece of gauze wrapped over the funnel the ether was dripped. There was no alternative. There were no adequate resuscitative measures available except from R.A.F. sources. If we needed saline, plasma, or blood we had to supply them and the transfusion set. The local saline was so pyrogenic that it was unusable. Oxygen cylinders were supplied, but as there were no workable masks they were of limited value. Sterilization of all dressings was carried out by the R.A.F.

Ancillary medical services were elementary. A workable $x$-ray unit was only fitted some weeks before we left. Pathological services were limited to the most simple investigations, and more complicated procedures were carried out by the R.A.F. Practically all the public-health aspect of the work was done by the R.A.F. laboratory.

The control of endogenous diseases, considering the primitive sewage system and appalling housing conditions, appeared to be of little avail. Amoebiasis, for example, was widespread and tuberculosis common. Owing to the very limited accommodation available only a small number of cases of the latter disease likely to respond to conservative treatment were hospitalized. Advanced cases spread the disease.

We have made no attempt to list all the inadequacies-space does not permit-and while appreciating that some good has been done by the Colonial Medical Service in Aden nevertheless the general impression created is more than disappointing. Plans, we believe, have been prepared for some time for building a new hospital, but it should be obvious that many defections are unconnected with buildings. In concluding it is worth remembering that Aden is a "shop window" for Great Britain, and that a great many nationalities pass through the port. We felt that no poorer advertisement than the existing medical arrangements could be offered.-We are, etc.,

$$
\text { D. P. VAN MEURS, }
$$$$
\text { W. D. WYLIE, }
$$

Belmont, Surrey. Late Wing Commanders, R.A.F.V.R

\section{Pulmonary Embolism}

SIR,-I welcome Mr. Reginald S. Murley's criticism (Feb. 28 , p. 415) of my article "The Aftermath of Gastrectomy," which was of course no more than a brief summary of a huge subject. Even so I should have devoted more than six lines to an aftermath as serious as pulmonary embolism. However, I am not totally ignorant of modern views on the subject regarding the importance of deep peripheral thrombosis. When $\mathrm{Mr}$. Murley says that my statement, "Thrombosis usually arises in the abdominal wall, or, as in two of these cases, from extension of the thrombosis of haemorrhoids . . ." is preposterous, I disclaim all responsibility and place it fairly and squarely on the shoulders of Nature, since these cases were proved by postmortem examinations which I personally witnessed-the clots being traced respectively into the azygos and iliac veins (in one a mass of clot still projected into the superior vena cava from the vena azygos major). Nature has an awkward habit of being "preposterous" and oft-times interferes most inconsiderately with our ideas.

I should like to add that as a house-surgeon I was brought up to believe in the value of post-operative leg massage and exercises, introduced in those far-off days by Rutherford Morison, and this practice has been followed throughout my surgical life. However, as the figures in my article reveal, I have not been able to avoid pulmonary embolism by such simple measures. The modern extension of this idea and the introduction of reliable anticoagulants will it is hoped reduce the incidence of this dire complication, but $I$ think I was right in saying that it still remains " a bugbear," which the O.E.D. gives as "an object of (needless) dread."-I am, etc.,

London, W.1.

NORMAN C. LAKE.

\section{Bone Conduction in Otosclerosis}

SIR,--The report in the Journal of Feb. 14 (p. 308) of a paper on bone conduction in otosclerosis, read by Dr. R. R. Woods at a meeting of the Section of Laryngology and Otology of the Royal Academy of Medicine in Ireland, focuses attention on the gain in bone conduction in the operated ear which occurs after the fenestration operation for otosclerosis.
In this connexion I would like to emphasize the considerable lengthening of bone conduction which may occur in the unoperated ear after a fenestration operation on the other ear. This lengthening of bone conduction is a matter of clinical importance, as the following case illustrates.

A man aged 47 consulted me for bilateral deafness getting steadily worse during the last three years. He complained of moderate buzzing noise in each ear. On testing the hearing of the right ear, conversational voice could not be heard distinctly at a distance greater than one-and-a-half feet; with the left ear loud conversation could only be heard occasionally at about one inch On testing bone conduction with a 256 tuning fork it was found to be lengthened by a few seconds on the right mastoid bone and to be reduced by about $50 \%$ on the left mastoid bone. The ear drums were normal in appearance and the eustachian tubes were freely patent.

As a result of the reduced bone conduction in the left ear it was thought that there was likely to be a considerable degree of nerve deafness in this ear. On the other hand, the findings with the right ear indicated that a good result to the fenestration operation on this ear was likely. A right fenestration operation was therefore performed. During the second week after this operation the hearing to conversational voice with the right ear improved to hearing at a distance of about twenty feet. At the same time hearing to conversational voice with the left ear improved to about one foot and bone conduction, using the same 256 tuning fork, was lengthened to normal for this fork.

In view of the improved bone conduction in the left ear it was decided to do a second fenestration operation on this ear. As there was very little giddiness this was done three-and-a-half weeks after the first operation. The patient began getting up four days after the second operation, as there was even less giddiness after this operation than after the first operation. During the second week after the operation the hearing with the left ear became as good as the hearing with the right ear

The patient was discharged from hospital six weeks after the first operation, and was back on duty as a commercial traveller two weeks later. It is now six weeks since he left hospital, and all tinnitus has subsided and he is hearing conversational voice at about twenty-four feet in each ear.

The lengthening of bone conduction described above is one manifestation of a syndrome which I call contralateral concomitant auditory facilitation of the fenestration operation. Usually in the less marked cases of bilateral deafness the syndrome is marked by considerable improvement in hearing to conversational voice by the unoperated ear.-I am, etc.,

$$
\text { London, W.1. }
$$

W. H. B. Magauran.

\section{Calciferol in Tuberculosis}

SIR,-Under dispensary conditions my experience with calciferol in tuberculous cervical adenitis has not been so satisfactory as that obtained by Dr. G. B. Dowling et al. (March 6, p. 430) treating cases in institutions. Over a period of four months seven out of eight cases given 100,000 units of calciferol daily by mouth showed a slow improvement which could easily have been due to the other general measures employed. In one case the glands broke down and operation for an abscess was required.

From this it would appear that once a case is under suitable conditions calciferol acts by giving a stimulus to the defence mechanism of the body. Such an action has been noted in six cases of advanced pulmonary tuberculosis. Three or four weekly intramuscular injections of 300,000 units of calciferol improved the general condition, reduced the degree of fever, and induced a gain in weight. No adverse toxic effects were seen in treating these cases of pulmonary tuberculosis.

One other case received $6,000,000$ units by injection in 20 days. There were no deleterious effects, nor was any improvement noticed.-I am, etc.

$$
\text { Wallasey. }
$$

\section{R. Grenville Mathers.}

\section{Retroperitoneal Haemorrhage in Pregnancy}

SIR,-Dr. J. Kenworthy Ogden's report of this case (Feb. 28, p. 389) is of great interest. It does not appear necessary to try to correlate the aetiology of retroperitoneal haematoma with toxaemia of pregnancy. The post-mortem findings confirmed the presence of massive spontaneous intraperitoneal haemorrhage with no definite bleeding point. The first reported case of this condition was by Barber in 1909 . The patient, aged 32, collapsed two days after labour. At operation no definite 
bleeding point was found. She recovered. There was no mention of toxaemia of pregnancy in this case.

In the absence of high blood pressure and arteriosclerosis the heart is incapable of raising the blood pressure sufficiently high to cause rupture of a normal artery, and some congenital or developmental defect must precede spontaneous rupture. Bruce (1937) suggested that the haemorrhage in this group may be due to rupture of a miliary aneurysm or a "junctional area." Bruce's suggestion was soon confirmed by Schuster (1937), who described a case of multiple aneurysms of the splenic artery which she believed were of congenital or developmental origin in a case of familial haemorrhage telangiectasis. Shallow, Herbut, and Wagner (1946) demonstrated defects in the muscle coat of the inferior pancreatico-duodenal artery associated with a ruptured congenital saccular aneurysm at the first bifurcation and three small fusiform dilatations in the more distal parts.

At post mortem it is often difficult to demonstrate the bleeding point in this type of massive spontaneous intraperitoneal haemorrhage. However, careful dissection of the affected part and sectioning of the neighbouring vessel will demonstrate the cause-a ruptured miliary aneurysm or congenital defect of the arterial wall.-We are, etc..

\section{Liverpool.}

A. C. BREWER.

R. MARCUS.

Barber, M: C. (1909). British Medical Journal, 2, 203.

Bruce, J. (1937). Lancet, 1, 1451.

Schuster, N. H. (1937). J.'Path. Bact., 44, 29.

Shallow, T. A., Herbut, P. A., and Wagner, F. B. (1946). Surgery, 19, 177.

\section{Cancer of the Lung}

SiR,-With reference to recent correspondence I wish to draw your attention to the fact that in a small research (confined to males) carried out here it was found that the incidence of cancer of the lung fell more heavily on smokers than nonsmokers-correction for age having been made. Unfortunately the number of cases investigated was too small to be statistically significant.

Our ignorance of the fundamental cause or causes of cancer is almost completely unrelieved. We know that in a small proportion of cases the inciting cause is the repeated application of a carcinogenic agent contained in tar, soot, oil, or other substance. The apparent increase in cancer has been almost entirely in cancers of inaccessible parts of the body.

Improved methods of diagnosis- of which radiology is the chief-are accountable in part for the greater frequency with which a diagnosis of cancer of the lung is now made. Also it is possible that more people develop cancer of the lung because more people live to the age at which cancer usually occurs. Gassing during the first world war and its sequelae apparently did not predispose to lung cancer.

Whether tars or other agents contained in tobacco act as carcinogenic agents in the lung is a disputed question. A complete statistical survey of smokers and non-smokers on this point has yet to be made. The quality and amount of tobacco consumed, the frequency of inhalation, age, sex, race, and all other relevant factors would have to be included in such an investigation.-I am, etc.,

Public Health Department, Halifax.

$$
\text { G. C. F. RoE. }
$$

\section{Intervertebral Disk Lesions}

SIR,-Dr. Michael Kelly (Feb. 28, p. 416) rightly draws attention to the well-known observation that pain is not a characteristic feature of lesions known to produce pressure on nerve trunks. As was shown by Lewis and Pochin, ${ }^{1}$ in nerve paralysis from pressure touch fails early, motor power next, " fast" pain later, and delayed pain last. Before pain sensation is lost transitory hyperalgesia may occur, so that the earliest signs of nerve pressure are alternating periods of numbness and cutaneous tingling in the area of distribution. Pain such as that of the sciatic syndrome does not occur.

On page 424 of the same issue, however, your adviser's reply under "Any Questions?" follows traditional thought in suggesting that the sciatic pain of disk lesions is due to pressure or stretching of nerve-roots. That may be so, but there is at least some evidence that other factors are involved. Kellgren and Lewis ${ }^{2}$ demonstrated that irritation of a deep somatic structure such as an interspinous ligament by injection of hypertonic saline resulted in pain throughout the corresponding sclerotome with accompanying muscle spasm. We can aver from personal experience and self-experiment that the pain produced by so stimulating the fifth lumbar interspinous ligament is clinically and subjectively indistinguishable from that occurring spontaneously in an L5-S1 disk lesion.

Now, primarily, herniation of the nucleus pulposus is a joint lesion which produces strains on surrounding ligamentous structures and may finally give rise to nerve-root or spinal compression. Such a joint disorder might reasonably be expected to produce pain throughout the corresponding sclerotome, irrespective of whether or not nerve pressure occurs. In fact, a large proportion of patients in whom the diagnosis is subsequently confirmed by operation never present any acceptable objective evidence of nerve pressure such as muscle weakness, anaesthesia, or loss of deep reflexes.

Before the phenomena are clearly understood two facts must be explained. (1) Application of a short plaster corset, even without preliminary extension, may rapidly relieve pain, although the area of anaesthesia persists or, as in one case under our observation, actually increases. Clearly such benefit cannot be due to relief from nerve pressure, but it may well be the result of immobilizing, at least partly, the grossly deranged spinal joint and thereby diminishing the deep segmental pain arising therefrom. (2) We have found that in many cases of undoubted disk prolapse temporary relief from pain follows injection of the corresponding interspinous ligament with $1 \%$ procaine solution. This cannot be the result of relief from nerve pressure though the change may be explicable upon the basis of the theory recently propounded by Cohen.s

The understanding of the pain mechanisms involved in these cases is a matter of some importance. In most instances the primary necessity of treatment is the relief from pain, a problem as yet incompletely solved. Fuller understanding of the manner in which pain is produced will surely pave the way to more universally satisfactory results,

It is generally accepted that on clinical evidence alone the level of a lumbar spine disk lesion cannot be confidently predicted. It may be that the present conception of sciatic pain as the result of root compression is responsible for this difficulty, and if it were regarded as a deep segmental pain due to joint disturbance this difficulty might be resolved. Oversimplified views of the pain mechanism in this condition are liable to follow a mechanical interpretation of the functions of the central nervous system.

The problem is a complex one and, clearly, many questions remain to be answered. We are at present engaged in an investigation of this subject and hope to communicate our findings in due course.-We are, etc.,

London, W.2.

J. Donaldson Craig. A. W. LIPMANN-KeSSEL.

\section{REFERENCES}

1 Clin. Scl., 1938, 3, 141.

3 Lancet, 1947, 2, 933

\section{The Extent of Neurosis}

SIR,-Stimulated by the correspondence on this subject 1 decided to classify the patients I saw during a period of twelve weeks-from Nov. 9, 1947, to Jan. 31, 1948. Some of the facts and figures emerging may be of interest. The practice under review is a large mixed private and panel practice in a town of some 25,000 inhabitants, extending also to some of the neighbouring villages. It is run by four partners, who share the work as equally as possible, apart from the fact that nearly all the anaesthetics are done by one partner, and a very large proportion of the midwifery by another. This accounts for the rather disproportionately small showing of these two types of work in the figures following, since the work is that of one partner out of the four.

A total of 3,200 patients were seen ; of these 1,744 were surgery attendances, 1,074 were visits to patients in their own homes, and 382 to patients in the local cottage hospital. 2,241 were "old patients," while 959 were first attendances.

Among the acts of service were included: minor surgery, 43; injections, 82; anaesthetics, 3 ; obstetric attendances, 43 (including 16 antenatal and 4 post-natal examinations); life insurance, 6; examination for entrance to Civil Service, etc., 10.

Not included in the total of acts of service above was the dispensing of medicine on 656 occasions and the making of 50 telephone calls on behalf of patients. Furthermore, the paper work in the course of this period, apart from record-keeping, included the writing of 1,034 prescriptions and the issuing of 879 certificates of various kinds, as well as the notifying of 21 cases of infectious 